\title{
Evaluation of Serum Level of 8-Isoprostane in Patients with Acne Vulgaris
}

A.M.El-Sheikh ${ }^{1}$, H.H.Sabry ${ }^{1}$, A.I.Mostafa ${ }^{1}$ and W.A.A.E-lHaleem ${ }^{2}$

${ }^{1}$ Dermatology,Venereology and Andrology Dept., Faculty of Medicine, Benha Univ., Benha, Egypt

${ }^{2}$ Clinical and Chemical pathology Dept., Faculty of Medicine, Benha Univ., Benha, Egypt

E-Mail:amalelsheikh24@gmail.com

\begin{abstract}
Skin break out Vulgaris is perhaps the most widely recognized constant incendiary skin illnesses. It basically influences juvenile and some of the time proceeds into adulthood, however may introduce at whatever stage in life. For most patients, skin inflammation is described by the presence of comedones, papules, pustules and knobs. Lamentably, it might prompt perpetual distorting scars. It principally influences the face, neck, upper trunk and upper arms. Skin break out is a multifactorial sickness in which a few elements have been ensnared, including; hormonal impacts, follicular hyperkeratinization, and multiplication of P.acnes, provocative, natural and hereditary components. Responsive oxygen flavors and lipid peroxidation assume a job in the inception and movement of epithelial irritation in the pilosebaceous unit. In skin break out, sebum organization is changed. Lipid peroxidation of unsaturated fats creates both intracellular and extracellular ROS. The age of ROS can modify the consistency and arrangement of sebum. Isoprostanes are a gathering of prostaglandin-like mixes that are created by the response of free extremists with arachidonic corrosive, incorporating arachidonic esters in phospholipids. Creation of isoprostanes is very much recorded to increment in direct extent to the degree of oxidative pressure. Point : The point of current examination was to explore the relationship between serum level of 8-Isoprostane, and danger of AV among Egyptian patients. Technique: The current examination included 30 patients experiencing AV, notwithstanding 30 obviously solid people of coordinated age and sex as control gathering. All were researched for 8-Isoprostane level and thought about. 8Isoprstane level was assessed utilizing monetarily accessible ELISA packs. End: From the aftereffects of present investigation, it is finished up 8-Isoprstane level is higher in skin break out vulgaris patients in corrospnding to the infection seriousness.
\end{abstract}

Key words: Acne vulgaris, Oxidative stress, 8-Isoprostane.

\section{Introduction}

Skin inflammation is assessed to influence $9.4 \%$ of the worldwide populace, making it the eighth most pervasive sickness worldwide [1]. It principally influences juvenile and at times proceeds into adulthood, however may introduce at whatever stage in life. For most patients, skin inflammation is portrayed by the presence of comedones, papules, pustules and knobs. Lamentably, it might prompt perpetual distorting scars. It essentially influences the face, neck, upper trunk and upper arms [2]. Acne is a multifactorial infection in which a few components have been ensnared, including [3].

1. Propionibacterium acnes which is respon $\neg$ sible for the creation of proinflammatory go betweens by the insusceptible framework

2. Sebaceous organ hypersecretion of sebum.

3. Hyperkeratosisfollowed by check of the follicle.

4. Inflammatory elements delivered by the skin and invulnerable framework.

Contemplates have zeroed in on the fundamental connection between oxidative pressure and the pathology of skin inflammation vulgaris [4] . It is accepted that an im $\neg$ balance in the creation of oxygeninferred supportive of oxidants, otherwise called responsive oxygen species (ROS), and cell limit of antiᄀoxidant safeguard, most likely prompts oxidative pressure marvel and growth of ef $\neg$ fects of ROS [5].

Addtionally, connections among ROS and lipids bring about polyunsaturated unsaturated fat peroxidation and creation of harmful aldehydes, for example, malondialdehyde (MDA), which can be utilized as biomarkers to assess lipid peroxidation in cells [6] .Out of totally oxidized fattyacids ,the best pointers of oxidative pressure in vivo are right now theF2-isoprostanes, explicitly 8-isoprostane which has been concentrated as a biomarker of lipid peroxidation in numerous examinations and estimation of its fixation in blood or pee is a grounded strategy for the demonstrative evaluation of oxidative stress [7]. The connection between serum level of 8 -isoprostane and skin infections with oxidative pressure had been examined eg: in psoriasis [8] , oral lichen planus [9] and fundamental sclerosis [10], however not explored in skin inflammation vulgaris.

\section{Materials and methods}

\subsection{The Study Population}

This is a case-control study. The ebb and flow study had been led after the endorsement by Research Committee at Faculty of Medicine, Benha University. The examination included 30 subjects that were chosen from those going to the Dermatology Outpatient's Clinic at Benha University Hospital, Faculty of Medicine, Benha University, Qalyobia, Egypt. Preceding commencement of the investigation, each subject was educated about the point of the examination and an educated assent was gotten from every person before test assortment. This current investigation included: Patient gathering included 30 skin inflammation vulgaris patients. Likewise, 30 clearly sound, age and sex coordinated people included as a benchmark group. 


\subsection{ELISA assays of 8-isoprostane}

Quantitative determinations of serum 8-Isoprostane were achieved using a double-antibody sandwich Enzyme Linked Immune Sorbent Assay technique (ELISA) was used to detect serum level of 8Isoprostane using a commercial Human 8-Isoprostane ELISA Kit for research use only.

\subsection{Statistical analysis}

The collected data was revised, coded, tabulated and introduced to a PC using Statistical package for Social Science (IBM Corp. Released 2011. IBM SPSS Statistics for Windows, Version 20.0. Armonk, NY: IBM Corp.). Data were presented and suitable analysis was done according to the type of data obtained for each parameter.Student $\mathrm{T}$ Test was used to assess the statistical significance of the difference between two study group means. For the comparison of the three groups' means, one way analysis of variance (ANOVA) was used. Chi-Square test was used to examine the relationship between two qualitative variables. Fisher's exact test: was used to examine the relationship between two qualitative variables when the expected count is less than 5 in more than $20 \%$ of cells. Correlation analysis: To assess the strength of association between two quantitative variables. The correlation coefficient defines the strength and direction of the linear relationship between two variables. $p$ is significant if $<0.05$ at confidence interval $95 \%$.

\section{Results}

Mean disease duration was 2.1 years \pm 0.6 years.

Clinical assessment of studied patients revealed that; 15 of patients $(50 \%)$ had gradual onset of AV and $15(50 \%)$ had progressive course of AV. The course was stationary in $(36 \%)$ of patients and progressive in $(64 \%)$ of the patients.

\subsection{Biochemical assessments of 8-isoprostane}

The mean 8-Isoprostane serum levels were significantly higher in patient group than their controls Table (1) .

Table (1) Comparison between patient group and their controls regarding serum level of 8-Isoprostane (ng/dl).

\begin{tabular}{lcccc}
\hline & & $\begin{array}{c}\text { Acne Vulgaris } \\
\mathbf{N}=\mathbf{3 0}\end{array}$ & $\begin{array}{c}\text { Control } \\
\mathbf{N}=\mathbf{3 0}\end{array}$ & $\mathbf{P}$ \\
\hline Serum & Mean士 SD & $101.2 \pm 29.2$ & $30.4 \pm 6.9$ & $0.004^{\mathrm{T}}$ \\
$\begin{array}{l}\text { 8Isoprostane } \\
(\text { ng/dl) }\end{array}$ & Median & $4.8-694.3$ & $2-137.9$ & \\
\hline
\end{tabular}

\section{Discussion}

This case-controlled examination was intended to survey serum levels of 8-Isoprostane in AV patients and connect their levels with the seriousness of the sickness.

In the current investigation, there was factually critical expansion in 8-Isoprostane (ng/dl) level among patients bunch than their controls, additionally it is essentially expanding with expanded skin break out seriousness. This was in concurrence with that acquired by [8] who expressed that the oxidative pressure measures assume a significant job in psoriasis etiopathogenesis and indicated that Increased serum and lesional 8-isoprostane levels were seen in psoriatic patients contrasted and the sound controls. Another examination by [11] indicated that correlation of SOD levels in the patient gathering as per the seriousness evaluations of skin inflammation (mellow, moderate, and extreme) demonstrated that there was a critical contrast in SOD levels in the three evaluations, being lower in serious cases and higher in gentle cases $(\mathrm{P}<$ $0.001)$. In the interim, examination of MDA levels in various skin inflammation seriousness grades demonstrated that there was a huge distinction between the three evaluations. MDA levels were essentially lower in gentle cases and higher in extreme cases $(\mathrm{P}<$ 0.001).

\section{Conclusion}

From the aftereffects of present examination, it is reasoned that serum level of 8-Isoprostane can be utilized for early conclusion of $\mathrm{AV}$, showing seriousness of the sickness or utilized as a prognostic factor for advancement of post skin inflammation scars.

\section{Recommendation}

The aftereffects of our examination should be deciphered considering its impediments, as the current investigation incorporated a generally little example size. Further examinations are expected to explore the exact systems by which 8-Isoprostane add to the pathogenesis and seriousness of skin inflammation vulgaris.

\section{References}

[1] A.L.Zaenglein, A.L.Pathy, B.J.Schlosser. Guidelines of care for the management of acne vulgaris. J Am Acad Dermatol, Vol.74(5), PP.945-973,2018.

[2] D.D.Lynn, T.Umari, C.A.Dunnick . The epidemiology of acne vulgaris in late adolescence. Adolesc Health Med Ther, Vol.7, PP.13-25,2016.

[3] T.X.Cong, D.Hao, X.Wen. From pathogenesis of acne vulgaris to anti-acne agents. Arch Dermatol Res, Vol.311, PP.337-349,2019.

[4] T.Zhu, F.Fang, D.Sun. Piceatannol Inhibits P. acnes-Induced Keratinocyte Proliferation and Migration by Downregulating Oxidative Stress 
and the Inflammatory Response. Inflammation, Vol.43(1), PP.347-357,2020.

[5] H.A.Al-Shobaili. Oxidants and anti-oxidants status in acne vulgaris patients with varying severity. Ann Clin Lab Sci, Vol.44(2), PP.202207,2014.

[6] G.L.Milne, Q.Dai , II. LJ.Roberts. The isoprostanes-25 years later. BBAMOL Cell Biol L, Vol.1851(4), PP.433445. ,2015.

[7] T.J.Van't Erve, M.B.Kadiiska, S.London . Classifying oxidative stress by F2-isoprostane levels across human diseases: a metaanalysis. Redox Biol, Vol.12, PP.582-599,2017.

[8] X.Jiao, Z.Guo, T.Chen. Determination of antioxidant capacity and 8-iso-prostaglandin F $2 \alpha$ levels in patients with psoriasis and their significance. Chin J Dermatol, Vol.45(6), PP.388-391,2012.

[9] M.Amirchaghmaghi, S.I.Hashemy, B. Alirezaei. Evaluation of plasma isoprostane in patients with oral lichen planus. J Dent , Vol.17(1), PP.2125,2016.

[10] K.Bourji, A.Meyer, E.Chatelus . High reactive oxygen species in fibrotic and nonfibrotic skin of patients with diffuse cutaneous systemic sclerosis. Free Radic Biol Med, Vol.87, PP.282289, 2015.

[11] Y.F.El Garem, R.A.Ahmed, M.A.Ragab . Study of oxidative stress in different clinical severities of acne vulgaris. Egypt J Dermatol Venerol, Vol.34(1), PP.53-57,2014. 